\title{
GABAergic Inhibition Sharpens the Frequency Tuning and Enhances Phase Locking in Chicken Nucleus Magnocellularis Neurons
}

\author{
Iwao Fukui, ${ }^{1,2}$ R. Michael Burger, ${ }^{2,3}$ Harunori Ohmori, ${ }^{1}$ and Edwin W Rubel ${ }^{2}$ \\ ${ }^{1}$ Department of Physiology and Neurobiology, Faculty of Medicine, Kyoto University, Kyoto, 606-8501, Japan, ${ }^{2}$ Virginia Merrill Bloedel Hearing \\ Research Center, University of Washington, Seattle, Washington 98195-7923, and ${ }^{3}$ Department of Biological Sciences, Lehigh University, \\ Bethlehem, Pennsylvania 18015
}

\begin{abstract}
GABAergic modulation of activity in avian cochlear nucleus neurons has been studied extensively in vitro. However, how this modulation actually influences processing in vivo is not known. We investigated responses of chicken nucleus magnocellularis (NM) neurons to sound while pharmacologically manipulating the inhibitory input from the superior olivary nucleus (SON). SON receives excitatory inputs from nucleus angularis (NA) and nucleus laminaris (NL), and provides GABAergic inputs to NM, NA, NL, and putatively to the contralateral SON. Results from single-unit extracellular recordings from 2 to 4 weeks posthatch chickens show that firing rates of auditory nerve fibers increased monotonically with sound intensity, while that of NM neurons saturated or even decreased at moderate or loud sound levels. Blocking GABAergic input with local application of TTX into the SON induced an increase in firing rate of ipsilateral $\mathrm{NM}$, while that of the contralateral NM decreased at high sound levels. Moreover, local application of bicuculline to NM also increased the firing rate of NM neurons at high sound levels, reduced phase locking, and broadened the frequency-tuning properties of NM neurons. Following application of DNQX, clear evidence of inhibition was observed. Furthermore, the inhibition was tuned to a broader frequency range than the excitatory response areas. We conclude that GABAergic inhibition from SON has at least three physiological influences on the activity of NM neurons: it regulates the firing activity of NM units in a sound-level-dependent manner; it improves phase selectivity; and it sharpens frequency tuning of NM neuronal responses.
\end{abstract}

\section{Introduction}

Sounds are encoded as a train of action potentials in auditory nerve fibers (ANFs), and various stimulus features are represented by these patterns of activity. In birds, ANFs bifurcate to innervate two cochlear nuclei, nucleus magnocellularis (NM) and nucleus angularis (NA), where further processing occurs. $\mathrm{NM}$ is specialized for transmission of phase information bilaterally to nucleus laminaris (NL), where the interaural phase differences are first processed (Young and Rubel, 1983; Carr and Konishi, 1990; Overholt et al., 1992). NM is specialized both morphologically and physiologically. First, large ANF presynaptic terminals, called the end bulbs of Held (Parks and Rubel, 1978; Whitehead and Morest, 1981; Jhaveri and Morest, 1982a,b,c), are formed on somas of NM neurons and generate a large postsynaptic current that can minimize jitter in synaptic transmission (Brenowitz and Trussell, 2001b). Second, low-voltage-activated

Received March 21, 2010; revised July 27, 2010; accepted July 30, 2010.

This work was supported by Grants-in-Aid for Scientific Research (KAKENHI) from the Ministry of Education, Culture, Sports, Science, and Technology of Japan (20700349 to I.F. and 20220008 to H.O.), a grant-in-aid from Fujiwara Memorial Foundation to I.F., and National Institutes of Health/National Institute on Deafness and Other Communication Disorders Grants DC00466 to R.M.B. and DC003829 and DC04661 to E.WR. We appreciate Dr. Leonard M. Kitzes, two anonymous reviewers and the editor for valuable comments and suggestions.

Correspondence should be addressed to Iwao Fukui, Department of Physiology and Neurobiology, Faculty of Medicine, Kyoto University, Kyoto, 606-8501, Japan. E-mail: fukui@nbiol.med.kyoto-u.ac.jp.

DOI:10.1523/JNEUROSCI.1484-10.2010

Copyright $\odot 2010$ the authors $\quad$ 0270-6474/10/3012075-09\$15.00/0 potassium channels are robustly expressed in the postsynaptic membrane, which decreases the membrane resistance and correspondingly shortens the membrane time constant (Reyes et al., 1994; Rathouz and Trussell, 1998). These and other specialized features of postsynaptic membrane and the morphological features of synaptic terminals are differentially expressed along tonotopic axis in the NM (Fukui and Ohmori, 2004).

The superior olivary nucleus (SON) is known to receive excitatory inputs from the ipsilateral NA and NL and is thought to receive inhibitory inputs from the contralateral SON. It sends GABAergic projections to the ipsilateral NM, NA, NL, and contralateral SON (Lachica et al., 1994; Westerberg and Schwarz, 1995; Burger et al., 2005b), and constitutes a major source of GABAergic inputs to these targets. In brainstem slice experiments, the GABAergic activity decreases the membrane resistance of NM and NL neurons (Yang et al., 1999; Monsivais et al., 2000). Models propose SON functions to improve ITD processing in two primary ways: (1) equalizing the binaural cochlear nucleus activity (Burger et al., 2005b; Dasika et al., 2005), and (2) improving the phase sensitivity in NM by shortening the membrane time constant (Yang et al., 1999; Monsivais et al., 2000). Until now, it has been unclear how this unusual depolarizing GABAergic input actually functions in vivo, especially considering that it must overcome the very large excitatory currents generated by the secure end-bulb synapses of ANF terminals to suppress activity (Brenowitz and Trussell, 2001a). We investi- 
gated the processing of auditory information in vivo to further understand the role of this inhibitory system on sound-evoked activity in NM. Our results support three main conclusions regarding inhibitory function. First, SON regulates the firing rate of NM at moderate and high sound pressure levels (SPLs), stimulus conditions for which ANF inputs are expected to be depressed. Second, inhibition improves the phase selectivity of high-frequency NM neurons. Finally, GABAergic input sharpens frequency tuning of NM neurons. These three related consequences of SON input have important implications for the computation of ITDs.

\section{Materials and Methods}

In vivo neuron recording and measurement. We used 128 young adult chickens aged $15-31 \mathrm{~d}$ (P15-P31). Chickens were anesthetized using chloral hydrate $(150 \mathrm{mg} / \mathrm{kg})$ and urethane $(1 \mathrm{~g} / \mathrm{kg})$ or, in some cases, only urethane $(1 \mathrm{~g} / \mathrm{kg})$. Supplemental doses of urethane were administered, if the subjects showed any sign of discomfort. Animals were maintained at $40^{\circ} \mathrm{C}$ with a heating pad. Sound stimuli were presented through a pair of hollow ear bars with a closed acoustic system from a pair of earphones (EF-1935, Knowles Electronics). The sound pressure level was calibrated at the beginning of each experiment. All experiments were made in a sound-isolation booth placed within a soundproof, shielded room. The detailed surgical and physiological recording methods were previously described (Fukui et al., 2006) except as noted below. In experiments using the multibarrel electrodes or the application of 6,7-dinitroquinoxaline-2,3-dione (DNQX) to the brainstem, the surface of NM was exposed by aspiration of the cerebellum leaving the cerebellar peduncle. Neural responses were recorded with custom-written data acquisition software (B. Warren, University of Washington, Seattle, WA) or software written in MATLAB (Fukui et al., 2006). After recordings, the brainstem was fixed by transcardiac perfusion with $10 \%$ formalin and sliced at 100 $\mu \mathrm{m}$ to document the electrode tracts. The source of 1518 units recorded in NM was identified as either an ANF or a NM unit by the waveform of spike-triggered average (STA) in response to white noise stimulus (supplemental Fig. $1 \mathrm{Bb}$, available at www.jneurosci.org as supplemental material) (ANF, $n=628$; NM, $n=844$ ) and/or the latency to click stimuli (supplemental Fig. $1 A$, available at www.jneurosci.org as supplemental material) (ANF, $n=130 ; \mathrm{NM}, n=120$ ). Supplemental Figure $1 \mathrm{Ba}$ (available at www.jneurosci.org as supplemental material) shows the example of the STA measurement. The zero crossing time (arrowheads) between the second and the third significant peaks was stable regardless of the sound level. This time value was used as one criterion to distinguish ANF from NM units. SON units were also confirmed by the electrode tract as well as the latency of STA and/or from the latency to the click stimulus (supplemental Fig. 1C, available at www.jneurosci.org as supplemental material).

Quartz glass electrodes (\#QF100-70-10, Sutter Instrument) filled with $1 \mathrm{~m}$ sodium chloride were used for extracellular single-unit recordings. After detection of single-unit activity by white noise sound (90 dB), characteristic frequency (CF) was determined as previously described (Fukui et al., 2006). The firing rate-intensity function was measured with CF tones at randomized levels (from 10 to $90 \mathrm{~dB}$ SPL, $10 \mathrm{~dB}$ steps) (Fig. $1 A$ ). The sound duration was 50 or $70 \mathrm{~ms}$ with a $3 \mathrm{~ms}$ rise and fall time for a sinusoidal envelope and the interstimulus interval was typically $500 \mathrm{~ms}$. Firing rate was measured by counting spikes in a window from $3 \mathrm{~ms}$ after stimulus onset to the end of sound stimulus for $>3$ trials. The criterion for a threshold increase in spike rate was defined as the spontaneous firing rate plus 40 spikes/s (Warchol and Dallos, 1990). Maximal firing rate was defined as the maximal firing rate for the CF sound of $10-90 \mathrm{~dB}$ SPL. A depression index was computed as the ratio of the firing rate to a
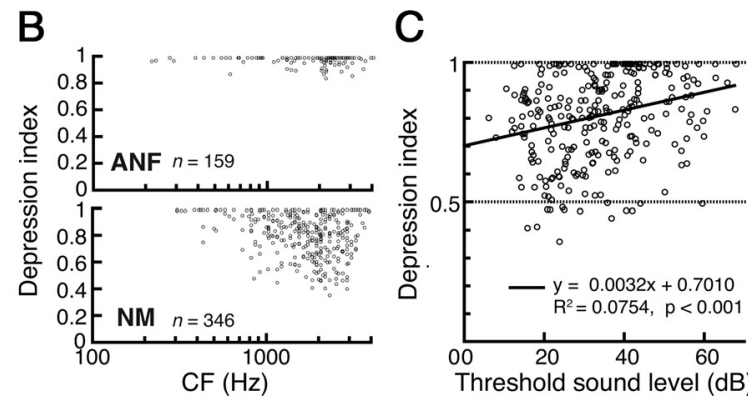

Figure 1. Difference in the rate-intensity relationship between ANF and NM. $A$, The average of firing rate for ANF (open circles, $n=127$ ) and NM (filled circles, $n=304$ ) units, of (F higher than $1 \mathrm{kHz}$. Although the firing rate of ANF increased monotonically with the sound intensity, the firing rate of NM was saturated around $60 \mathrm{~dB}$ SPL and was often reduced at higher sound levels. similar $C F$ neurons in NM. C. The threshold sound level for NM units having $C F>1 \mathrm{kHz}$ was plotted against depression index. The depression index was weakly dependent on the threshold sound level.

CF tone presented at $90 \mathrm{~dB}$ SPL to the maximal firing rate of the unit at any intensity. Therefore, a high depression index value $(\sim 1.0)$ indicates little or no spike suppression, while lower values indicate stronger spike suppression. Frequency tuning was determined by using a series of stimuli in which we varied frequency by $1 / 12$ or $1 / 6$ octave steps and intensity levels from below threshold to $90 \mathrm{~dB}$ SPL. Frequency selectivity was defined as the frequency range evoking above threshold firing rates at each intensity. Inhibitory frequency range was defined as the frequency range evoking firing rates falling two SDs below the spontaneous rate. The ratio of sensitive frequency $(H / L)$ was defined as the ratio of the high end of the frequency range evoking a response divided by the lower limit of the frequency range at a given intensity. Vector strength (VS) (Goldberg and Brown, 1969) was calculated from $15 \mathrm{~ms}$ after the sound onset to the end of sound stimulus (see Figs. 5, 6) as follows:

$$
\mathrm{VS}=\sqrt{\left[\sum \sin \left(\alpha_{i}\right)\right]^{2}+\left[\sum \cos \left(\alpha_{i}\right)\right]^{2}} \div N,
$$

where $a_{i}$ is the phase position of a single spike and $N$ is the total number of spikes. The phase angle of the stimulus sinusoid at which each spike occurs is expressed as a vector of unit length. The vectors of all spikes are then averaged with the mean length indicating the vector strength. Thus, this measure equates to the relative abundance of firing at a particular phase angle. Vector strength varies from 0 (no phase locking) to 1 (perfect phase locking). Data are presented as mean $\pm \operatorname{SEM}(n=$ number of samples). Statistical analysis was performed by the unpaired or paired Student's $t$ test or ANOVA. Removal of cerebellum did not affect the firing properties (spontaneous firing rate, maximal firing rate, threshold, depression ratio) of ANF and NM units (supplemental Fig. 2, available at www.jneurosci.org as supplemental material) $(p>0.45)$.

Application of TTX to SON. Tetrodotoxin (TTX) was iontophoretically applied to SON using a glass electrode of $\sim 40 \mu \mathrm{m}$ tip diameter. SON was located by the evoked potential in response to a $50 \mathrm{~ms}$ white noise stimulus. The electrode was filled with artificial CSF (ACSF) containing 0.5 mM TTX and $1 \mathrm{mg} / \mathrm{ml}$ Alexa-568-conjugated $10 \mathrm{kDa}$ dextran amine. TTX and the dye were injected by applying a large current $( \pm 40 \mathrm{nA}, 1 \mathrm{~s}$ positive and $1 \mathrm{~s}$ negative, $20 \mathrm{~min}$ ). After the injection of TTX, the evoked potential response along the dorsal to ventral extent of the SON to white noise was almost completely suppressed. The injection site was confirmed by Alexa-568 following unit recordings (supplemental Fig. 3, available at www.jneurosci.org as supplemental material). TTX was successfully injected into SON for 7 chickens (P17-P24). Single-unit recordings were made from $30 \mathrm{~min}$ to $5 \mathrm{~h}$ after the injection of TTX.

Injection of bicuculline in NM. Multibarrel "piggyback" electrodes, composed of a single sharp electrode (borosilicate: AM SYSTEMS, Cat\#602000, or quartz: (\#QF100-70-10, Sutter Instrument) glued to a 
A



B

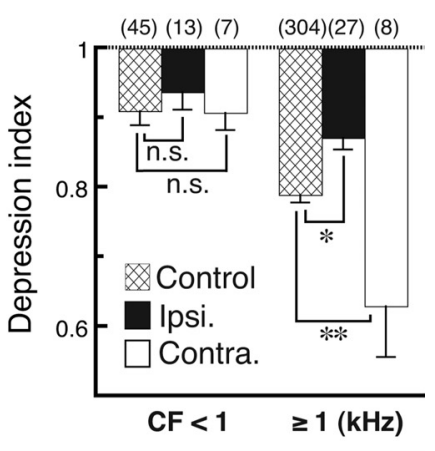

there was substantial variation in the depression index between units of similar $\mathrm{CF}$, the depression index of NM units depended on the CF of units. Depression of spike rate was seen primarily in the middle to high-CF NM units (Fig. $1 B$ ), in contrast to units with $\mathrm{CFs}<1 \mathrm{kHz}$. Consistent with these findings, as described below, the influence of inhibition (or SON input) was most clearly observed in neurons with CFs above $1 \mathrm{kHz}$. Furthermore, there was a weak, but significant relationship between threshold and firing rate depression at high sound levels; units having low acoustic thresholds tended to have low depression indexes (larger depression) (Fig. 1C).

To test the influence of the SON in $\mathrm{NM}$, the activity of the SON was blocked by local iontophoretic injection of $0.5 \mathrm{~mm}$ TTX, leaving the contralateral SON intact (supplemental Fig. 3, available at www.

three or five barrel pulled glass pipette (AM SYSTEMS, Cat\#609000, or World Precision Instruments, 5B120F-4), were used for iontophoretic application of bicuculline methiodide to NM (Havey and Caspary, 1980). The tip of the multibarrel electrode was broken to make the total tip diameter of $\sim 25-40 \mu \mathrm{m}$ and the sharp recording electrode was glued using cyanoacrylate glue and self-curing resin. The tip of the single sharp electrode protruded $20-30 \mu \mathrm{m}$ from the tip of the multibarrel electrode. Internal solution of $1 \mathrm{~m} \mathrm{NaCl}$ was used for the single sharp electrode. Internal solution of $19.6 \mathrm{~mm}$ bicuculline in $154 \mathrm{~mm} \mathrm{NaCl}$ at $\mathrm{pH} 3$ and control solution (154 mM NaCl, $\mathrm{pH} 3$ ) was used for the multibarrel electrode. The center barrel of the electrode was used as a current sink to balance the total injected current $(154 \mathrm{~mm} \mathrm{NaCl}, \mathrm{pH}$ 7). A retention current $(6 \mathrm{nA})$ was always applied during the insertion of electrode through the brainstem. The amplitude of the injected current ranged between -40 and $-80 \mathrm{nA}$. The injection of control solution (154 mm $\mathrm{NaCl}, \mathrm{pH} 3$ ) had no effect on NM unit responses ( $n=2$, data not shown).

Application of DNQX onto the brainstem. ACSF containing (in mм: 120 $\mathrm{NaCl}, 2.5 \mathrm{KCl}, 2 \mathrm{CaCl}_{2}, 1 \mathrm{MgCl}_{2}, 26 \mathrm{NaHCO}_{3}, 1.25 \mathrm{NaH}_{2} \mathrm{PO}_{4}, 17$ glucose) or ACSF including $20 \mu \mathrm{M} \mathrm{DNQX}$ at $40^{\circ} \mathrm{C}$ ) was continuously perfused on the surface of brainstem by a peristaltic pump (2-4 $\mathrm{ml} / \mathrm{min})$ in 10 chickens (P21-P27). The peristaltic pump was also used to recover the ACSF solution. To stabilize the effects of drug, unit recordings were made after $>30$ min from the beginning of drug perfusion.

\section{Results}

\section{Firing properties of ANF and NM units and block of} SON activity

Figure $1 A$ shows the averaged rate-intensity function for ANF and NM units when a CF tone stimulus was applied. The spontaneous firing rate of NM units was significantly higher than for ANF units (NM, $101.9 \pm 2.1$ spikes/s, $n=413$; ANF, $48.7 \pm 2.1$ spikes/s, $n=201, p<0.0001)$. The spontaneous firing rate, the threshold sound level (NM, 31.34 $\pm 0.7 \mathrm{~dB}$; ANF, $30.3 \pm 1.0 \mathrm{~dB})$ and maximal firing rate (NM, $260.9 \pm 4.2$ spikes/s; ANF, $370.0 \pm$ 7.9 spikes/s) were comparable to those of previous reports (Warchol and Dallos, 1990; Salvi et al., 1992; Fukui et al., 2006). The firing rate of ANF units increased with the sound level and the rateintensity relationship was monotonic in almost all units recorded. In contrast, the firing rate of NM units reached a maximum at an intermediate sound level and was slightly decreased or saturated at higher input levels (ANF). The depression index was defined as the firing rate at $90 \mathrm{~dB}$ SPL divided by the maximum firing rate of a unit. Thus low values indicate strong suppression. The depression index was lower than 0.95 in $72.7 \%$ of NM units, and only $6.7 \%$ of ANF units. Interestingly, although jneurosci.org as supplemental material), and the responses of NM neurons were tested for 7 chickens. The depression of the NM units ipsilateral to the TTX inactivated SON was reduced compared to that seen in control NM neurons and, particularly, that observed in contralateral NM units (Fig. 2A). The effect of inactivating the SON input was only apparent at high intensities (above $\sim 60 \mathrm{~dB}$ ) and was much more dramatic for neurons with high-frequency CFs than low-CF units. Moreover, the depression of the ipsilateral NM after the TTX injection to SON was significantly smaller than that of the control, while that of the contralateral NM after the TTX injection was larger than that of the control for units with CFs above $1 \mathrm{kHz}$ (Fig. 2B). ANOVA followed by individual comparisons revealed no significant differences for units with CFs $<1 \mathrm{kHz}$. For units with $\mathrm{CFs} \geq 1 \mathrm{kHz}$, depression at high stimulus levels was significantly less on the ipsilateral side than controls $(p<0.05)$ and reliably greater on the contralateral side than controls $(p<0.001)$. Similarly, the difference between the average depression index ipsilateral and contralateral to the TTX blockade of SON activity was highly significant $(p<0.001)$. This difference between ipsilateral and contralateral depression index was not due to differences in $\mathrm{CF}$ distribution for the two populations of neurons. The means and SDs did not differ significantly $[<1 \mathrm{kHz}$ : ipsilateral $\mathrm{CF}=767.2 \pm$ $48.7 \mathrm{~Hz}(n=13)$, contralateral $\mathrm{CF}=616.5 \pm 81.7 \mathrm{~Hz}(n=7) ; \geq 1$ $\mathrm{kHz}$ : ipsilateral $\mathrm{CF}=1745.0 \pm 129.7 \mathrm{~Hz}(n=27)$, contralateral $\mathrm{CF}=1619.3 \pm 192.9 \mathrm{~Hz}(n=8)]$.

There was no significant difference in the spontaneous rate in $\mathrm{NM}$ (ipsilateral $127 \pm 4.7$ spikes/s, $n=40$; contralateral $131.7 \pm 9.3$ spikes/s, $n=15 ; p=0.62$ ) or the threshold sound level (ipsilateral $20.0 \pm 1.2 \mathrm{~dB}, n=40$; contralateral $20.3 \pm 2.7 \mathrm{~dB}, n=15 ; p=0.91)$. However, the NM spontaneous rates were higher for the TTXtreated population than for the control data reported in Figure 1 (control 103.2 \pm 2.3 spikes/s, $n=346$; TTX 128.3 $\pm 9.3, n=55, p<$ 0.01 ). The maximal firing rate was not significantly different between three groups for $\mathrm{CFs}<1 \mathrm{kHz}$ (control, ipsilateral, contralateral; ANOVA, $p=0.13$ ). For CFs $\geq 1 \mathrm{kHz}$, the mean maximum rate of ipsilateral TTX-treated NM neurons $(347.8 \pm 10.3$ spikes/s, $n=$ 27) was significantly higher than control ( $274.6 \pm 3.7$ spikes/s, $n=$ $304, p<0.001)$, but that of contralateral TTX treated was not (319.2 \pm 22.6 spikes/s, $n=8)$. ANF activity was not significantly influenced by TTX injection into SON (spontaneous rate: control $43.5 \pm 39.4$ spikes/s, $n=31$, + TTX $36.8 \pm 18.0$ spikes/s, $n=7, p=$ 
0.66; rate at $90 \mathrm{~dB}$ SPL: control $356.0 \pm 98.6$ spikes/s, $n=31$, +TTX $427.8 \pm 84.5$ spikes/s, $n=7, p=0.08$ ).

\section{Bicuculline facilitated the firing activity} of NM units

SON is known to make a GABAergic projection to NM. We further evaluated the effects of GABAergic inhibition by locally blocking $\mathrm{GABA}_{\mathrm{A}}$ receptors in $\mathrm{NM}$ with the iontophoretic application of bicuculline using a multibarrel piggy-back electrode while recording from NM neurons. Firing rates before, during, and after the application of bicuculline were measured. It should be stressed that this manipulation did not evaluate possible contributions of $\mathrm{GABA}_{\mathrm{B}}$ receptors to the firing properties of NM neurons, and it is known that $\mathrm{GABA}_{\mathrm{B}}$ receptors are found both presynaptically and postsynaptically in NM (Burger et al., 2005a). The results of blocking $\mathrm{GABA}_{\mathrm{A}}$ receptors for a typical neuron are shown in Figure 3. For this neuron, following 1-4 min of bicuculline iontophoresis $(-60 \mathrm{nA})$, the firing rate in response to a $90 \mathrm{~dB}$ CF tone gradually increased, and saturated within $6 \mathrm{~min}$ of bicuculline application (Fig. 3A). Again, the increase in the firing rate was observed only for stimuli above $\sim 60 \mathrm{~dB}$ SPL, and was not effective at either moderate $(50 \mathrm{~dB}$ SPL) or subthreshold (10 dB) levels (Fig. $3 A$, b in Fig. $3 B$ ). After $2-4$ min from the suspension of bicuculline application, the firing rate at $90 \mathrm{~dB}$ SPL decreased to the control rate, and no decrease was observed at 10 or $50 \mathrm{~dB}$ SPL (Fig. 3A). The complete rate-intensity function was similar to the control after 6-10 min of recovery from bicuculline application (c in Fig. 3B). In 24 NM neurons tested, a significant increase of the firing rate was observed at sound levels higher than $60 \mathrm{~dB}$ SPL and the mean firing rate at $90 \mathrm{~dB}$ SPL was increased $1.38 \pm 0.04$ times (Fig. 3C,Da), while the spontaneous firing rate was not significantly changed ( $p=0.73$ ) (Fig. 3C). Iontophoretic application of the $\mathrm{pH}$ adjusted isotonic vehicle solution $(154 \mathrm{~mm}$ $\mathrm{NaCl}, \mathrm{pH} 3.0)$ had no significant effect on the firing activity $(n=$ 2 , data not shown). The largest increases in the firing rate following bicuculline application were found in units with the lowest depression index values in the control $(p<0.001)$ (Fig. 3Db). The firing rate of ANF units was not affected by the application of bicuculline ( $n=2$, data not shown). Despite the large changes in firing rate at high sound levels, the overall firing pattern was only marginally changed.

Figure 4 shows one example of the PSTH in the control $(A a)$ and during the application of bicuculline $(A b)$, and the averaged difference of firing rate $(B)$. After application of bicuculline, the firing rate increased during the entire $80 \mathrm{~ms}$ CF tone stimulus with the exception of the onset response (Fig. 4, vertical dotted line). In this example, the increase became significant starting at $\sim 8 \mathrm{~ms}$ following stimulus onset and continued to be statistically significant until offset of the response to sound (Fig. $4 B$ ). The averaged increase in firing rate between 10 and 80 ms was $54.9 \pm$ 8.4 spikes/s to this $90 \mathrm{~dB}$ SPL CF stimulus. That corresponds to a $44.2 \%$ above the firing rate of before the bicuculline treatment
(124.3 \pm 9.9 spikes/s, $n=24)$. Figure $4, C$ and $D$, shows average firing rates for the early portion of the PSTH and sustained response portion, respectively, as a function of stimulus level for the control condition and with bicuculline treatment. It is clear that the bicuculline treatment dramatically enhances responses to high-intensity stimuli during the sustained response, but has little or no effect during the onset response. While PSTH analyses show little change in the overall pattern of response, analyses of the temporal precision in responses to tone stimuli show that it was dependent on GABAergic input. VS is a measure of the degree of phase selectivity of a response (Goldberg and Brown, 1969). Figure $5 A$ shows one example of period histograms of spikes in under control conditions and during bicuculline treatment. Phase locking was observed in the control with a VS of 0.46 . During bicuculline application the period histogram broadened and the VS fell to 0.33 , while the firing rate increased to 1.55 times the control at $90 \mathrm{~dB}$ SPL. Ratios of VS measured at $90 \mathrm{~dB}$ SPL in each condition are plotted in Figure $5 B$. The averaged ratio of VS at $90 \mathrm{~dB}$ SPL was $0.92 \pm 0.02$ for all units studied in both conditions $(n=24, p<0.001)$. Again, the decrease in VS was more striking for higher CF units $(r=-0.47, p<0.05)$. VS decreases were also level dependent; a priori comparisons were made between control and bicuculline condition at each intensity. At 70, 80 , and $90 \mathrm{~dB}$ stimulus levels, the difference was significant $(p<$ $0.05)$. At lower intensities, the small differences in VS ratios were not reliable (Fig. 5C).

\section{Inhibitory tuning curve in NM}

The acoustically evoked inhibition in NM, demonstrated by suppressing the excitatory synaptic input from ANFs, was frequency 

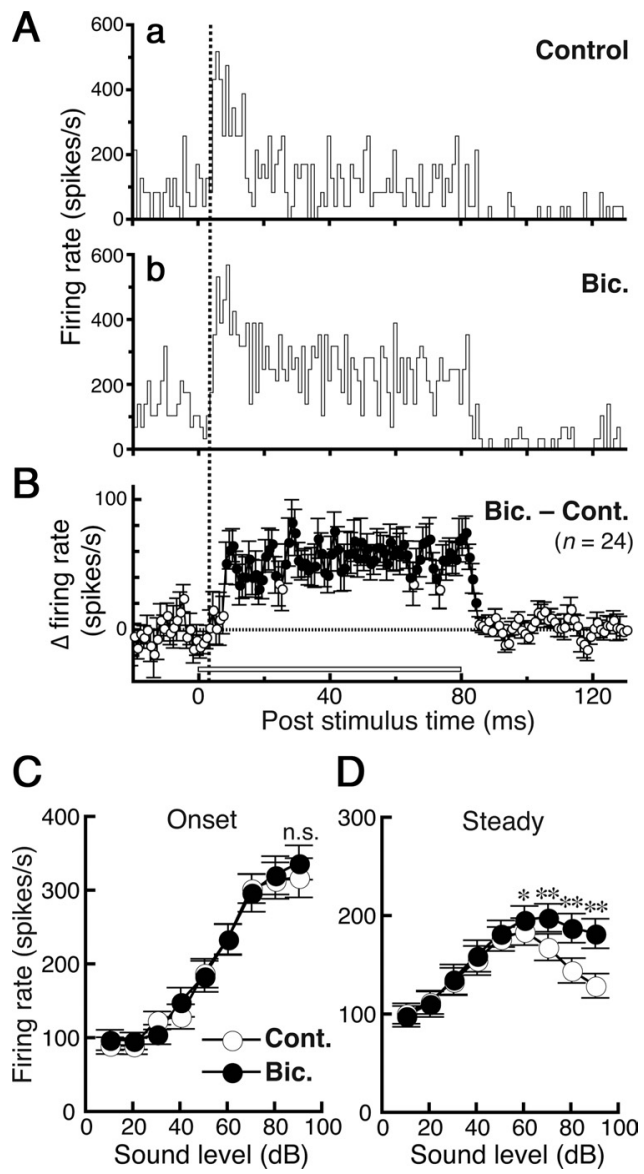

Figure 4. Time course of the bicuculline-sensitive response. $A$, PSTH of NM neuron for the control ( $\boldsymbol{a}$, average of 23 trials) and during the application of bicuculline ( $\boldsymbol{b}$, average of 28 trials). Sound level was $90 \mathrm{~dB}$ SPL at CF (1903 Hz) and the duration was $80 \mathrm{~ms}$ (open bar at the bottom in $\boldsymbol{B}$ ). $\boldsymbol{B}$, The averaged difference between in the control and in bicuculline ( $n=24$ ) was plotted by closed (significant, $p<0.05$ ) and open circles (not significant). The effect of bicuculline emerged with a delay of $\sim 5 \mathrm{~ms}$ to the onset of sound and was almost constant during the remainder of the sound stimulation. Bin width is $1 \mathrm{~ms}$ for $A$ and $B$. $C, D$, The averaged firing rates for the onset period $(0-8 \mathrm{~ms}, C)$ and sustained period $(8-80 \mathrm{~ms}, \boldsymbol{D})$. The significant increase was observed only with higher intensity stimuli of the sustained response. ${ }^{* *} p<0.01 ;{ }^{*} p<0.05 ;$ n.s., not significant. dependent (Fig. 6). We applied $20 \mu \mathrm{M}$ DNQX onto the surface of the brainstem to partially block AMPA receptors in NM from activation by ANFs. The spontaneous firing rate of NM units was slightly lower in the presence of DNQX than in control conditions (in DNQX, $97.5 \pm 4.2$ spikes/s, $n=58$; in controls, $116.5 \pm$ 6.2 spikes/s, $n=49, p<0.05$ ), while the spontaneous firing rates of ANF units was not significantly different (in DNQX, 52.6 \pm 3.6 spikes/s, $n=41$; in control, $49.7 \pm 4.7$ spikes/s, $n=35$ ). After the application of DNQX, the auditory-evoked discharges in NM units nearly ceased, only occurring at the onset of the sound stimulus for most neurons (Fig. 6Aa). Surprisingly, the ongoing firing rate for remainder of the duration of the tone decreased below the spontaneous level. The onset responses enabled us to identify NM units and characterize their frequency selectivity. The zero crossing time and latency in spike-triggered averaging (supplemental Fig. $1 B$, available at www.jneurosci.org as supplemental material) of the click response corresponded to that of untreated NM units (supplemental Fig. $1 A$, available at www. jneurosci.org as supplemental material).

The excitatory and inhibitory response tuning was assessed for each NM unit in two time windows with respect to the stimulus. The excitatory onset responses were assessed in a window including the initial $15 \mathrm{~ms}$, while the inhibitory response area was measured from the area of response suppression in the remaining 15-80 ms period (see Materials and Methods). The excitatory response exhibited a relatively narrow tuning range at $90 \mathrm{~dB}$ SPL (Fig. 6Ab) (the frequency range above the threshold level was $1.32-2.07 \mathrm{kHz}$ ) when compared to the inhibitory tuning range (Fig. 5Ab) (the frequency range below the inhibitory threshold level was $0.45-3.75 \mathrm{kHz}$ ). Figure $6 \mathrm{Ba}$ shows one example of the tuning curve for the excitation measured from the onset response and the tuning curve of the inhibition measured from the ongoing response. The tuning curve of the excitatory onset response was sharp. Figure $6 B b$ shows another example of the inhibitory tuning curve in the unit for which the onset excitatory response was not detectable. The tuning curve of the inhibitory response was again very broad (Fig. $6 \mathrm{Bb}$ ) and overlapped with that of the excitatory response at loud sound levels (Fig. $6 \mathrm{Ba}$ ). The $\mathrm{W}$-shape of inhibitory tuning curve in Figure $6 \mathrm{Ba}$ is likely due to the remaining excitatory response that canceled the inhibition during the sound stimulus.

The frequency ranges of the excitation and the inhibition are compared in Figure $7 A$ for DNQX-treated NM neurons $(n=30)$. For most units (24 of 30 units) the frequency range of the inhibition extended well beyond the frequency range of the excitation. In 3 of 30 neurons, the frequency range of the excitation could not be detected with the DNQX treatment. While a small onset response was detectable, it did not achieve our criterion for threshold across a range of frequencies. In contrast, evoked inhibition suppressed spontaneous activity in these neurons (top 3 units in Fig. $7 A$ ) across a broad range of frequencies. In two units (indicated by closed arrowheads in Fig. 7A), the inhibition extended only to the high-frequency side, and in one unit (indicated by gray arrowhead in Fig. 7A) the inhibition extended only to the low-frequency side.

Tuning width was assessed for units

using the sensitive frequency ratio $(H / L)$,
Figure 5. Phase sensitivity of NM response to sound with and without bicuculline. $\boldsymbol{A}$, One example of the period histogram of NM neuron in control (left) and in the presence of bicuculline (right) ( $C F=1.8 \mathrm{kHz}$ ). Spikes were counted from 20 trials of sound stimulation ( $90 \mathrm{~dB}$ SPL at CF) in control and 12 trials with bicuculline. $B$, Ratios of VS at $90 \mathrm{~dB}$ SPL with CF tone stimuli between the control and bicuculline conditions were calculated and plotted for all neurons. In the presence of bicuculline, the VS ratio was reduced, particularly in high-CF neurons. The correlation coefficient was $-0.47(p<0.05)$. $C$, The average of the VS ratio was plotted against sound level. VS was significantly decreased at intensities above $60 \mathrm{~dB}$ SPL $(p<0.05)$. Parentheses indicate the number of neurons. All calculations of VS ratio were made at the sound levels greater than threshold.
B

A
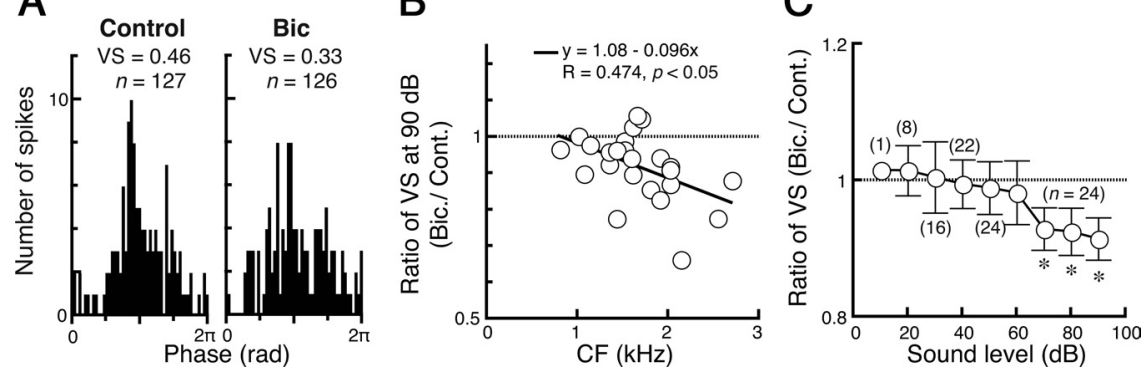
defined as the ratio of the high-frequency limit of the response range divided by the low-frequency limit. Population mean $H / L$ ratios for ANF, NM, and the excitatory and inhibitory response areas for NM units treated with DNQX are shown in Figure $7 B$. ANF and NM showed sharp frequency tuning. DNQX-treated NM units (NM-Exc. in Fig. 7B) also showed sharp excitatory tuning, but the inhibitory area (NM-Inh. in Fig. $7 B$ ) was strikingly broader. $H / L$ ratios for all four conditions were significantly different from each other (ANF $2.9 \pm 0.2$; NM $2.4 \pm 0.1$; NMExc. $1.8 \pm 0.1$; NM-Inh. $9.7 \pm 1.3$ ). The broad tuning of the inhibitory response area was similar in scale to the excitatory tuning curves observed in recordings of individual SON units (data not shown).

The $H / L$ of NM units was also CF dependent and had a tendency to be smaller than that of ANF units for CFs $>1 \mathrm{kHz}$ (Fig. $7 B$ ). The range of sensitive frequencies, defined by the maximum and minimum frequencies evoking suprathreshold responses at any intensity, was compared between the control and bicuculline conditions for a group of NM units. Figure $8 \mathrm{~A}$ shows one typical example. The range of sensitive frequency (bounded by two arrows) became broader by bicuculline. For almost all $11 \mathrm{NM}$ units studied in this way, the $H / L$ ratio became larger after the application of bicuculline (Fig. $8 B$ ). The average of $H / L$ was $1.8 \pm 0.3$ in control and $2.5 \pm 0.5$ in the bicuculline $(n=11)$. Bicuculline broadened the $H / L$ by $1.4 \pm$ $0.2(p<0.05)$. The average of the lower and higher bounds was $1154 \pm 185 \mathrm{~Hz}$ and $1653 \pm 127 \mathrm{~Hz}$ for the control, $1031 \pm$ $172 \mathrm{~Hz}$ and $1958 \pm 112 \mathrm{~Hz}$ for the bicuculline conditions. Both sides of bounds were significantly modified by the application of bicuculline $(p<0.01$ for lower bound, $p<0.05$ for higher bound). These results, together with data from Figure $7 B$, show that the tuning of excitation in NM while GABAergic input is blocked was much narrower than the tuning of inhibitory input while excitation was blocked.

\section{Discussion}

In this report, we evaluated the contribution of GABAergic input from the SON to processing of acoustic features in NM. We revealed at least three physiologically significant roles of GABAergic input to the NM in vivo. First, SON controls the firing rate of NM in a sound-level-dependent manner (Figs. 1-3). Second, the inhibition improves phase responsiveness in NM (Fig. 5). The improvement of the VS was more significant in high-CF units (Fig. 5B) and was significant only at the more intense sound levels (Fig. 5C). Third, the inhibition sharpens frequency tuning at NM (Fig. 8). In each case, the influence of GABAergic input was most prominent for cells responsive to higher frequencies $(>1$ $\mathrm{kHz}$ ) and for high or relatively high-intensity stimuli.

\section{Degree of the inhibition in NM}

The synaptic projection from SON to NM is known to be GABAergic (Lachica et al., 1994). The intracellular chloride concentration is elevated in mature NM neurons and the reversal potential of GABAergic IPSP is between $-25 \mathrm{mV}$ and $-37 \mathrm{mV}$ (Lu and Trussell, 2001; Monsivais and Rubel, 2001). As a result, GABAergic input depolarizes the membrane of NM neurons, yet the net effect of GABAergic input remains strongly inhibitory via two mechanisms. First, the depolarization reduces the membrane resistance by the activation of low-voltage-activated potassium channels and second, the kinetically slow depolarization causes threshold accommodation (Yang et al., 1999; Monsivais et al., 2000; Howard et al., 2007; Howard and Rubel, 2010). Our results are consistent with the above results from in vitro studies, showing that GABA indeed inhibits acoustically driven NM activity in vivo.

The excitatory input to NM has been well characterized by in vitro studies as well. The ANF terminals form large end bulb of Held synapses on NM neurons that individually generate very 
A NM (DNQX)

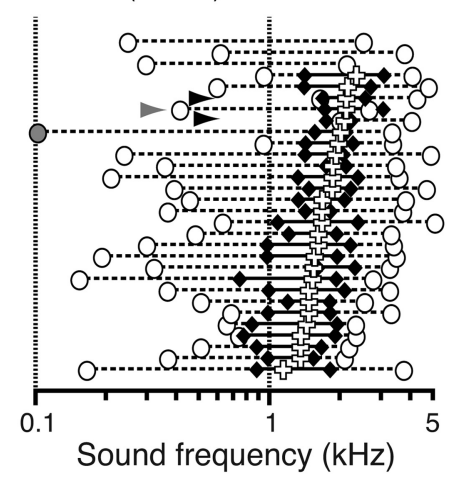

B

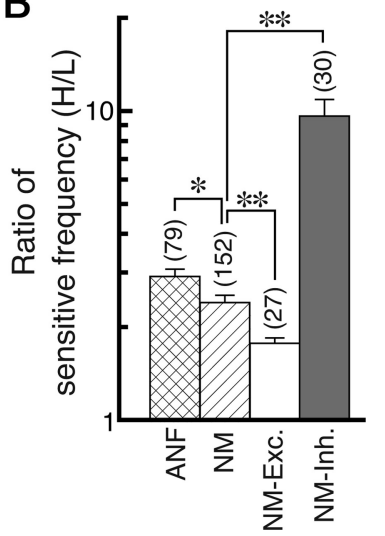

Figure 7. Inhibitory tuning curves are broader than excitatory tuning curves in NM neurons. $\boldsymbol{A}$, The inhibitory range (open circles) and excitatory range (closed diamonds) of NM neurons observed after the application of DNQX. Open crosses show the CF of the neuron. The inhibitory tuning was generally broader than the excitatory frequency range. The black arrowheads indicate two neurons for which inhibitory tuning was biased to the high-frequency side, while the gray arrowhead indicates a neurons with inhibition biased to the low-frequency side of the excitatory area. $B$, Population averages of the $H / L$ ratio of excitatory and inhibitory range for units of CF higher than $1 \mathrm{kHz}$. NM inhibition (NM-Inh.) and NM excitation (NM-Exc.) were measured from the data after the application of DNQX (Fig. $6 A$ ). The $H / L$ ratio of the inhibitory range of NM (NM-Inh.) was significantly broader than that of ANF (open circles) or NM (closed circles). The $H / L$ ratio of NM neurons were significantly narrower than ANF axons for neurons of CF higher than $1 \mathrm{kHz}$. In one case, a neuron had its lower limit of frequency sensitivity below 100 $\mathrm{Hz}$ (gray circle in $A$ ); for this cell, the lower bound was assumed to be $100 \mathrm{~Hz}$ and calculated. ${ }^{* *} p<0.01 ;{ }^{*} p<0.05 ;$ n.s., not significant.



B

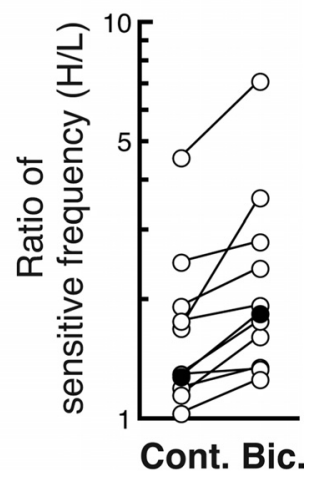

Figure 8. Bicuculline-sensitive current sharpened the frequency response. $\boldsymbol{A}$, The firing rate of an NM neuron ( $\mathrm{CF}=1510 \mathrm{~Hz}$ ) in the control condition (upper panel) and in the presence of bicuculline (lower panel). Dashed lines indicate the spontaneous firing rate and the thin solid horizontal lines indicate the threshold firing level. Arrows indicate the low and high bounds of the sensitive sound frequency. $\boldsymbol{B}$, The $H / L$ ratio was calculated for the control condition and in the presence of bicuculline. Data from each individual neuron are connected with a line. Black filled circles are data from the neuron shown in $\boldsymbol{A}$.

large synaptic currents sufficient to evoke an action potential in NM neurons in vitro (Parks and Rubel, 1978; Jhaveri and Morest, 1982a,b,c; Carr and Boudreau, 1991). However, at high firing rates, the ANF-to-NM synapse undergoes dramatic depression such that current amplitudes are reduced by $>80 \%$ at $>300 \mathrm{~Hz}$, even over brief input trains (Brenowitz and Trussell, 2001a). In this report, we demonstrate that acoustically evoked GABAergic input is sufficient to suppress excitatory tone responses of NM neurons in vivo. The inhibition was most efficacious during highintensity stimuli and in high-CF neurons. Indeed, blocking inhibition did not alter the spontaneous firing rate of NM neurons,

nor did it influence the evoked spike rate to low sound intensities. The bias in inhibitory efficacy toward high-CF neurons was surprising given that an earlier report showing stronger GABA immunoreactivity in the low-CF region in NM (Code et al., 1989). It may be noteworthy, however, that the quantitative analyses in this study used immunocytochemistry with a polyclonal antibody raised against GABA-gluteraldehyde conjugate. A later study by our group revealed significant differences in the patterns of labeling seen in NM with this antibody and an antibody to GAD-2, although the tonotopic gradient was not directly studied with the GAD-2 antibody (Code et al., 1990), and a subsequent study of the barn owl auditory pathways did not reveal a tonotopic gradient through NM (Carr et al., 1989). In addition, a qualitative review of tissue immunostained with modern antibodies against gephyrin, an intracellular marker for $\mathrm{GABA}_{\mathrm{A}}$ and glycine receptors, as well as GAD-65 (E. W Rubel, unpublished observations) failed to confirm the observations made by Code et al. (1989). Hence, the tonotopic gradient that was clearly observed in this early study may not have been specific to GABA per se, and this issue needs to be revisited by a combination of modern cell biological and physiological methods.

The dependence on CF and intensity may be due to a number of factors that control synaptic input magnitude and NM neuron excitability such as, synaptic depression (Brenowitz and Trussell, 2001a,b), and tonotopic variation in voltage-gated potassium channels (Fukui and Ohmori, 2004). These potassium channels are known to act cooperatively with GABAergic input to suppress spiking in these cells (Monsivais et al., 2000; Howard et al., 2007). Additionally, the number of ANF terminals impinging on NM neurons systematically decreases with CF (Fukui and Ohmori, 2004). Interestingly, the GABAergic inhibition fails to suppress responses to the putatively stronger excitatory synaptic inputs occurring at stimulus onset, when input from the SON may be relatively weak or delayed. Similar GABA-dependent spike suppression has been observed in primary-like anteroventral cochlear nucleus neurons in mammals (Caspary et al., 1994; Kopp-Scheinpflug et al., 2002; Gai and Carney, 2008).

In addition to CF and sound intensity, the GABAergic modulation of the NM neurons depended on depression index values. Among neurons of similar $\mathrm{CF}$, there was a large variation of depression values (Fig. $1 B$ ). This variation depends, at least in part, on the degree of inhibitory input across the population of NM neurons (Fig. 3Db); the firing rate of more depressed units tended to increase with bicuculline application. In addition, there was a weak, but reliable relationship between threshold and depression (Fig. 1C); low-threshold units tend to show higher depression indexes. These two, related, results probably account for the fact that the grand average of the firing rate was relatively flat at sound levels higher than $50 \mathrm{~dB}$ SPL (Fig. $1 \mathrm{~A}$ ), while the averaged depression index was 0.8 (Fig. $2 B$ ). Warchol and Dallos (1990) also report wide variation in threshold, saturation levels and degree of depression among NM units.

\section{Physiological significance of SON}

Inhibitory input provided by SON has emerged as a crucial component of sound localization circuitry in birds. First, Nishino et al. (2008) demonstrated that SON enhances the peak-trough contrast of ITD tuning especially in low-CF NL cells. SON also appears to influence the excitatory input to NL in several important ways.

In addition to the modulation of the ipsilateral NM as a function of intensity and CF, we show that the SON activity differentially modulates NM firing rates bilaterally, putatively via its 
GABAergic synaptic connectivity to the contralateral SON (Monsivais et al., 2000; Burger et al., 2005b). The physiological role of the mutual connectivity of the SONs has been proposed to equalize the binaural firing rate differences between each NM (Burger et al., 2005b, Dasika et al., 2005). Colburn and Durlach (1978) first proposed the computational advantage of equalization of input magnitude for coincidence detection. The complementary models proposed by Burger et al. (2005b) and Dasika et al. (2005) predicted that the SON-to-SON projection is itself inhibitory, and that higher activity in one SON would proportionally relieve the inhibitory feedback to the cochlear nucleus by suppressing activity in the contralateral SON. This hypothesis is supported by the demonstration that during TTX application to the SON, the firing rate of ipsilateral NM was facilitated, while the contralateral NM was depressed. These findings suggest that the reciprocal connectivity between the SONs (1) is mutually inhibitory and (2) functions to balance the magnitude of excitation to binaural coincidence detectors in NL. They further suggest that the SON contains a population of neurons that are driven by the ipsilateral ear and suppressed by the contralateral ear much like interaural level difference (ILD)-processing neurons in the mammalian lateral superior olive or in the avian dorsal lateral lemniscal nucleus (LLDp). LLDp encodes ILD by processing excitatory synaptic input from the ipsilateral NA and inhibition from contralateral LLDp (Manley et al., 1988; Konishi, 2003). Recently, work from our laboratories has reported that ILD-selective neurons are also found in the chicken SON (Coleman et al., 2010) and LLDp (Sato et al., 2010).

NM's bilateral output projects exclusively to NL, where coincidence-detecting neurons compute ITDs for sound localization, an essential feature of binaural hearing. Thus, NM's principal function is to provide accurate temporal information to NL neurons in the form of phase-locked discharges. The improvement of the phase locking by shortening the membrane time constant was predicted from in vitro studies (Yang et al., 1999; Monsivais et al., 2000). We show that GABAergic input does indeed improve phase locking in NM, especially in the high-CF neurons.

Finally, DNQX application directly to NM revealed inhibitory input that was more broadly tuned than the excitatory response area, suggesting that inhibition may suppress responses at and beyond the borders of an NM unit's frequency range, particularly at high sound levels. Enhancement of frequency contrast among inputs to NL through this mechanism might be particularly important under conditions of high background noise. Maintaining the frequency selectivity of each NM unit by SON would be advantageous for the processing of ITDs by reducing noisy, out-ofphase input from neurons phase locked to multiple sound frequencies.

In summary, localization of low-frequency sounds depends on elaborate circuitry that has evolved in birds and mammals for the precise computation of ITDs. This circuitry involves an elegant and highly specialized excitatory network known to preserve temporal information [for review, see Oertel (1997) and Trussell (1997)]. In addition, a large body of in vitro physiological and anatomical research over the past 20 years has led to important predictions about the functional significance of the inhibitory elements in this circuitry [for review, see Grothe (2003) and Burger and Rubel (2007)]. This report addresses several predictions made by these previous reports. GABAergic inhibition influences the rate, timing, and frequency tuning of NM neurons. In each case, this influence is predicted to result in improved temporal information provided by $\mathrm{NM}$ input neurons to coincidence-detecting neurons in NL. Thus, the inhibitory circuitry composed of the two coupled inhibitory feedback loops via the SON nuclei in the avian auditory brainstem appears to be critically important for the accuracy and precision in the neural computation of sound location.

\section{References}

Brenowitz S, Trussell LO (2001a) Maturation of synaptic transmission at end-bulb synapses of the cochlear nucleus. J Neurosci 21:9487-9498.

Brenowitz S, Trussell LO (2001b) Minimizing synaptic depression by control of release probability. J Neurosci 21:1857-1867.

Burger RM, Rubel EW (2007) Encoding of interaural timing for binaural hearing. In: The senses: a comprehensive reference, Vol 3 (Dallos P, Oertel D, eds), pp 613-630. Oxford: Elsevier.

Burger RM, Pfeiffer JD, Westrum LE, Bernard A, Rubel EW (2005a) Expression of $\mathrm{GABA}(\mathrm{B})$ receptor in the avian auditory brainstem: ontogeny, afferent deprivation, and ultrastructure. J Comp Neurol 489:11-22.

Burger RM, Cramer KS, Pfeiffer JD, Rubel EW (2005b) Avian superior olivary nucleus provides divergent inhibitory input to parallel auditory pathways. J Comp Neurol 481:6-18.

Carr CE, Boudreau RE (1991) Central projections of auditory nerve fibers in the barn owl. J Comp Neurol 314:306-318.

Carr CE, Konishi M (1990) A circuit for detection of interaural time differences in the brain stem of the barn owl. J Neurosci 10:3227-3246.

Carr CE, Fujita I, Konishi M (1989) Distribution of GABAergic neurons and terminals in the auditory system of the barn owl. J Comp Neurol 286:190-207.

Caspary DM, Backoff PM, Finlayson PG, Palombi PS (1994) Inhibitory inputs modulate discharge rate within receptive fields of anteroventral cochlear nucleus neurons. J Neurophysiol 72:2124-2233.

Code RA, Burd GD, Rubel EW (1989) Development of GABA immunoreactivity in brainstem auditory nuclei of the chick: ontogeny of gradients in terminal staining. J Comp Neurol 284:504-518.

Code RA, Durham D, Rubel EW (1990) Effects of cochlea removal on GABAergic terminals in nucleus magnocellularis of the chicken. J Comp Neurol 301:643-654.

Colburn HS, Durlach NI (1978) Models of binaural interaction. In: Handbook of perception (Carterette EC, Friedman M, eds), pp 467-518. New York: Academic.

Coleman WL, Fischl MJ, Trause DA, Burger RM (2010) Physiological heterogeneity of in the avian superior olivary nucleus. Abstracts of the thirtythird annual midwinter meeting of the Association for Research in Otolaryngology, Anaheim, CA.

Dasika VK, White JA, Carney LH, Colburn HS (2005) Effects of inhibitory feedback in a network model of avian brain stem. J Neurophysiol 94:400-414.

Fukui I, Ohmori H (2004) Tonotopic gradients of membrane and synaptic properties for neurons of the chicken nucleus magnocellularis. J Neurosci 24:7514-7523

Fukui I, Sato T, Ohmori H (2006) Improvement of phase information at low sound frequency in nucleus magnocellularis of the chicken. J Neurophysiol 96:633-641.

Gai Y, Carney LH (2008) Influence of inhibitory inputs on rate and timing of responses in the anteroventral cochlear nucleus. J Neurophysiol 99:1077-1095.

Goldberg JM, Brown PB (1969) Response of binaural neurons of dog superior olivary complex to dichotic tonal stimuli: some physiological mechanisms of sound localization. J Neurophysiol 32:613-636.

Grothe B (2003) New roles for synaptic inhibition in sound localization. Nat Rev Neurosci 4:540-550.

Havey DC, Caspary DM (1980) A simple technique for constructing 'piggyback' multibarrel microelectrodes. Electroencephalogr Clin Neurophysiol 48:249-251.

Howard MA, Rubel EW (2010) Dynamic spike thresholds during synaptic integration preserve and enhance temporal response properties in the avian cochlear nucleus. J Neurosci 30:12063-12074.

Howard MA, Burger RM, Rubel EW (2007) A developmental switch to GABAergic inhibition dependent on increases in Kvl-type $\mathrm{K}^{+}$currents. J Neurosci 27:2112-2123.

Jhaveri S, Morest DK (1982a) Neuronal architecture in nucleus magnocellularis of the chicken auditory system with observations on nucleus laminaris: a light and electron microscope study. Neuroscience 7:809-836. 
Jhaveri S, Morest DK (1982b) Sequential alterations of neuronal architecture in nucleus magnocellularis of the developing chicken: a Golgi study. Neuroscience 7:837-853.

Jhaveri S, Morest DK (1982c) Sequential alterations of neuronal architecture in nucleus magnocellularis of the developing chicken: an electron microscope study. Neuroscience 7:855-870.

Konishi M (2003) Coding of auditory space. Annu Rev Neurosci 26:31-55.

Kopp-Scheinpflug C, Dehmel S, Dörrscheidt GJ, Rübsamen R (2002) Interaction of excitation and inhibition in anteroventral cochlear nucleus neurons that receive large endbulb synaptic endings. J Neurosci 22:11004-11018.

Lachica EA, Rübsamen R, Rubel EW (1994) GABAergic terminals in nucleus magnocellularis and laminaris originate from the superior olivary nucleus. J Comp Neurol 348:403-418.

Lu T, Trussell LO (2001) Mixed excitatory and inhibitory GABA-mediated transmission in chick cochlear nucleus. J Physiol 535:125-131.

Manley GA, Köppl C, Konishi M (1988) A neural map of interaural intensity differences in the brain stem of the barn owl. J Neurosci 8:2665-2676.

Monsivais P, Rubel EW (2001) Accommodation enhances depolarizing inhibition in central neurons. J Neurosci 21:7823-7830.

Monsivais P, Yang L, Rubel EW (2000) GABAergic inhibition in nucleus magnocellularis: implications for phase locking in the avian auditory brainstem. J Neurosci 20:2954-2963.

Nishino E, Yamada R, Kuba H, Hioki H, Furuta T, Kaneko T, Ohmori H (2008) Sound-intensity-dependent compensation for the small interaural time difference cue for sound source localization. J Neurosci 28:7153-7164.

Oertel D (1997) Encoding of timing in the brain stem auditory nuclei of vertebrates. Neuron 19:959-962.
Overholt EM, Rubel EW, Hyson RL (1992) A circuit for coding interaural time differences in the chick brainstem. J Neurosci 12:1698-1708.

Parks TN, Rubel EW (1978) Organization and development of the brain stem auditory nuclei of the chicken: primary afferent projections. J Comp Neurol 180:439-448.

Rathouz M, Trussell L (1998) Characterization of outward currents in neurons of the avian nucleus magnocellularis. J Neurophysiol 80:2824-2835.

Reyes AD, Rubel EW, Spain WJ (1994) Membrane properties underlying the firing of neurons in the avian cochlear nucleus. J Neurosci 14:5352-5364.

Salvi RJ, Saunders SS, Powers NL, Boettcher FA (1992) Discharge patterns of cochlear ganglion neurons in the chicken. J Comp Physiol A 170:227-241.

Sato T, Fukui I, Ohmori H (2010) Interaural phase difference modulates the neural activity in the nucleus angularis and improves the processing of level difference cue in the lateral lemniscal nucleus in the chicken. Neurosci Res 66:198-212.

Trussell LO (1997) Cellular mechanisms for preservation of timing in central auditory pathways. Curr Opin Neurobiol 7:487-492.

Warchol ME, Dallos P (1990) Neural coding in the chick cochlear nucleus. J Comp Physiol A 166:721-734.

Westerberg BD, Schwarz DW (1995) Connections of the superior olive in the chicken. J Otolaryngol 24:20-30.

Whitehead MC, Morest DK (1981) Dual populations of efferent and afferent cochlear axons in the chicken. Neuroscience 6:2351-2365.

Yang L, Monsivais P, Rubel EW (1999) The superior olivary nucleus and its influence on nucleus laminaris: a source of inhibitory feedback for coincidence detection in the avian auditory brainstem. J Neurosci 19:23132325.

Young SR, Rubel EW (1983) Frequency-specific projections of individual neurons in chick brainstem auditory nuclei. J Neurosci 3:1373-1378. 